\title{
Supracondylar Fractures: A retrospective chart review comparing infection rate, antibiotic use, surgical time and cost of full surgical preparation and draping vs "semi-sterile" technique
}

\section{lan Laxdal ( $\nabla$ umlaxdai@myumanitoba.ca )}

University of Manitoba Neil John Maclean Health Sciences Libraries https://orcid.org/0000-00024899-955X

\section{Kevin Stockwell}

University of Manitoba Neil John Maclean Health Sciences Libraries

\section{Mark Xu}

University of Manitoba Neil John Maclean Health Sciences Libraries Jon Tan

University of Manitoba Neil John Maclean Health Sciences Libraries

\section{Sheila McRae}

Pan Am Clinic

\section{Paul Jellicoe}

University of Manitoba Neil John Maclean Health Sciences Libraries

Research article

Keywords: supracondylar, fracture, infections, pediatric, trauma, cost

Posted Date: July 14th, 2020

DOI: https://doi.org/10.21203/rs.3.rs-37322/v1

License: (9) This work is licensed under a Creative Commons Attribution 4.0 International License. Read Full License

Version of Record: A version of this preprint was published at Orthopedic Research and Reviews on December 1st, 2020. See the published version at https://doi.org/10.2147/ORR.S268517. 


\section{Abstract}

Background: Semi-sterile and full preparation and draping techniques are commonly used in closed reduction percutaneous pinning (CRPP) of supracondylar fractures. Debate exists whether full preparation and draping is safer than semi-sterile technique in regards to infection risk and the utility of pre-operative antibiotics. This study is a comparison of infection rates, pre-operative antibiotic administration, cost and surgical time between techniques.

Methods: A retrospective chart review of 336 pediatric patients with supracondylar fractures repaired with CRPP at our institution was completed between January 2014 and April 2018, 168 per technique. Infection rates, pre-operative antibiotic administration, preparation-to-incision time and cost in semi-sterile draping versus full preparation and draping techniques were compared.

Results: Of the 336 patients, $1 / 168(0.1 \%)$ in the full preparation and draping group developed an infection compared to $0 / 168(0 \%)$ patients in the semi-sterile group. Pre-operative antibiotics (Cefazolin) were administered to $76 / 168(23 \%)$ patients in the full preparation and draping group and $0 / 168(0 \%)$ in the semi-sterile group. The infection found received pre-operative antibiotics. Mean preparation-toincision time for the semi-sterile group was $2.4 \pm 2.0$ minutes and the full preparation and draping group was $9.9 \pm 4.2$ minutes $(p<0.001)$. Surgical supply cost was $\$ 80.72$ [CDN] and 108.24\$ [CDN], respectively, for the semi-sterile and full preparation and draping groups.

Conclusion: Risk of infection using a semi-sterile draping technique was safe and comparable to a full preparation and draping technique when used in CRPP of supracondylar fractures. The administration of pre-operative antibiotics does not appear to make a difference in infection rates. Semi-sterile operative technique is cost effective and has decreased preparation-to-incision time.

\section{Background}

Semi-sterile and full preparation and draping techniques are commonly used in closed reduction percutaneous pinning (CRPP) of supracondylar fractures. Current literature estimates infection rates between $0-7.3 \%{ }^{1-11}$ in CRPP of supracondylar fractures. lobst et al. ${ }^{4}$ in a series of 304 patients found zero infections with CRRP of supracondylar fractures using semi-sterile technique whereas Turgut et al. ${ }^{2}$ has recently shown that infection may be a matter of concern with $7.3 \%$ infection rates in the semi-sterile technique. Debate still exists among surgeons whether full preparation and draping is safer than semisterile technique regarding infection risk. Broadly, pre-operative antibiotics are used by some surgeons and not by others. Note that lobst et al. ${ }^{4}$ in their series of 304 patients found peri-operative antibiotics may not be necessary as $68 \%$ of their patients did not receive antibiotics in the perioperative or postoperative period resulting in zero infections. Surgical time and cost of semi-sterile and full preparation and draping techniques are not well reported in the literature. The current retrospective study was therefore set up to compare infection rates, pre-operative antibiotic administration, preparation-toincision time and cost in semi-sterile draping technique versus full preparation and draping in CRPP of 
supracondylar fractures. In the current study the main hypothesis was that there would be no significant differences of infection rates in semi-sterile compared to full preparation and draping techniques.

\section{Methods}

A retrospective review of medical records was performed for all patients aged 16 years of age or younger, who underwent CRPP of a supracondylar fracture at our institution between January 2014 and April 2018. Inclusion criteria were as follows: supracondylar fracture classified as either type 2 or 3 in the Gartland classification system ${ }^{12}$ surgically repaired with CRPP; K-wires (kirschner wires) left external to the skin; patients aged 0 to 16 years and complete follow-up for visits at one- and three-weeks postoperative. Exclusion criteria were as follows: open fractures; patients converted to open reduction; additional ipsilateral arm fractures; immunocompromised patients and those without complete follow-up.

Age, sex, fracture type according to the Gartland classification system ${ }^{12}$, pre-operative antibiotic administration, operative preparation-to-incision time, number of k-wires, medial k-wire use, infectious and mechanical complications were recorded.

The primary outcome of infection was defined as any patient prescribed antibiotics for clinical signs of infection within 28 days after surgery. All pins were removed within this time.

Operative care involved either semi-sterile or full preparation and draping techniques. All patients treated in the operative room with full preparation and draping technique went through the same presurgical and surgical preparation of the arm. All patients were placed in the supine position with the upper limb laid on an arm table. Splinting was removed, pre-operative cleansing of the entire arm was carried out using $4 \%$ Chlorhexidine Gluconate ("BD E-Z Scrub 747"). The arm was then prepped and draped in the typical fashion using Chlorhexidine Gluconate $2 \%$ w/v and Isopropyl Alcohol 70\% v/v Solution ("ChloraPrep* One-Step"). Depending on surgeon's preference, patients were either administered a dose of pre-operative antibiotics or not. Fractures were reduced with fluoroscopy and then stabilized with $2 \mathrm{~mm} \mathrm{~K}$-wires inserted laterally with percutaneous pinning. If a medial $\mathrm{K}$-wire was used this was done last with a miniopen approach. Number of K-wires was determined using the Gartland classification system ${ }^{12}$; 2 K-wires used for type 2, $3 \mathrm{~K}$-wires used for type 3 and a 4th wire added if required for stability. Once satisfactory stability was achieved K-wires were bent and then cut short using wire cutters. The wound was then bandaged with paraffin gauze dressing ("Jelonet; Smith \& Nephew"). Soft-roll was applied with a long arm plaster back slab which was kept in place until follow up. Post-operative antibiotics were not administered and patients did not receive any additional pin site care. All surgeons using full preparation and draping technique used the same protocol.

All patients treated in the operative room with semi-sterile technique went through the same presurgical and surgical preparation of the arm. Initial splinting was removed and cleansing of the arm was carried out in the same fashion as the full preparation and draping patients. No pre-operative antibiotics were used. Fractures were reduced with fluoroscopy with an assistant holding the arm in the reduced position, 
the surgeon put on sterile gloves, placed sterile towels around the surgical field, prepared the anticipated insertion site with Chlorhexidine Gluconate $2 \% \mathrm{w} / \mathrm{v}$ and Isopropyl Alcohol $4 \% \mathrm{v} / \mathrm{v}$ Solution ("STANHEXIDINE"). The fracture was stabilized with k-wires in similar fashion to the full preparation and draping patients. Number of K-wires was again determined by the Gartland classification system ${ }^{12}$, wires were bent and cut with the dressing and splinting applied in the same fashion as the full preparation and draping group. Post-operative antibiotics were not administered and patients did not receive any additional pin site care. All surgeons using semi-sterile technique used the same protocol.

For both groups, patients were first seen 7-10 days after surgery to check for radiographic signs of displacement. Plaster back-slabs were removed, K-wire sites inspected and long arm cylindrical fiberglass casts were applied. Patients were then seen again 21-28 days after surgery to remove the long arm cast and $\mathrm{K}$-wires as well as radiographic imaging. Follow up visits were then scheduled at 3 months, 6 months and 1 years after surgery (depending on surgeon preference).

To detect a difference in infection of $5 \%$ (deemed clinically significant by the authors) with a two-sided $5 \%$ significance level and a power of $80 \%$, a sample size of 168 patients per group was necessary. Descriptive statistics were generated for both groups and independent t-tests (continuous data) or chisquared tests (categorical data) were performed to ensure groups were comparable.

\section{Results}

An electronic search was conducted and 358 patient charts were screened with 336 consecutive eligible patients included in the study, 168 per group (Figure 1). Table 1 presents a description of each group. Of the 336 patients treated surgically, $1 / 168(0.1 \%)$ in the full preparation and draping group and 0/168 (0\%) patients in the semi-sterile group developed an infection requiring antibiotics. The affected patient developed an Enterobacter Cloacae septic arthritis of the elbow and was treated in hospital with irrigation debridement and IV antibiotics. This patient had full clinical recovery. Pre-operative antibiotics (cefazolin) were administered in this case.

Mean preparation-to-incision time for the semi-sterile group was $2.37 \pm 1.98$ minutes and for the full preparation and draping group was $9.85 \pm 4.24$ minutes which was statistically different $(p<0.001)$. Preoperative antibiotics (Cefazolin) were given to 76/168 (23\%) patients in the full preparation and draping group and 0/168 (0\%) in the semi-sterile group.

Complications other than infection are presented in Table 1. These complications included early pin removal and revision due to loss of reduction. Relative risk for complications versus no complications between the semi-sterile and full preparation and draping groups respectively $(5 / 168$ compared to $3 / 168)$ was $1.67(95 \% \mathrm{Cl} 0.40$ to $6.86, \mathrm{p}=0.50)$.

Costs for surgical supplies for the semi-sterile approach were estimated at $\$ 80.72$ [CDN] compared to $108.24 \$[C D N]$ for the full preparation and draping group. This resulted in a cost savings of $27.52 \$[C D N]$ per case when using semi-sterile technique. 


\section{Discussion}

The objective of this study was to compare infection rate, antibiotic administration, preparation-toincision time and cost of full surgical preparation and draping vs semi-sterile technique in CRPP of supracondylar fractures. There was no difference in infection rate between approaches, with only one patient having a reported infection across both groups, supporting our hypothesis. Cost savings were estimated to be approximately \$28 [CDN] per case when using semi-sterile technique over full preparation and draping, and a decreased preparation-to-incision time was also identified, translating to shorter overall surgical time.

The rate of infection found in the current study using the semi-sterile technique were similar to the findings of lobst et al. ${ }^{4}$. This study also supported the assertion in the current study that patients likely do not need pre-operative antibiotics administration. In contrast, Turgut et al. ${ }^{2}$ recently found high pin tract infection rates of $7.3 \%$ in a semi-sterile technique. In this large study, 712 patients had CRPP of supracondylar fractures with the semi-sterile technique of which $52(7 \%)$ developed pin tract infections. Infection was not well defined and patients were asked to visit their family doctor for pin site care every 4 days until they were removed. Kao et al. ${ }^{13}$ reported on a series of 61 patients treated for elbow fractures finding that infection rates were significantly higher in patients who had daily pin-site care then in patients who did not. This could explain the higher infection rates seen in Turgut et al. ${ }^{2}$ compared with lobst et al. ${ }^{4}$ and the current series.

A comparison of infection rates based on placement of pins including crossed or lateral pin fixation in 56 participants was reported by Shamsuddin et al. ${ }^{14}$ and found the rates were comparable. The current study was based on the use of lateral pins almost exclusively, but the findings of Shamsuddin et al. ${ }^{14}$ may support that the findings may extend to medial pins. However, this would need to be verified in further study.

Parikh et al. ${ }^{15}$ found that the risk of intracapsular pin placement using parallel lateral pins was found to be greater than either crossed or divergent lateral configurations. It is of interest that parallel lateral pins were used in the only infection found in our series with an associated septic arthritis of the elbow. This may suggest that the use of crossed or divergent lateral pins may reduce cases of septic arthritis.

The sole infection in the current study series was caused by Enterobacter cloacae which is a clinically significant gram-negative, anaerobic, rod-shaped bacterium. This bacterium is commensal in the enteric flora since they are found in soil and sewage, as well as in the human gastrointestinal tract. ${ }^{16}$ The authors suggest this infection may have been from hematogenous spread or inadequate hand-hygiene of surgical staff.

Surgical time and cost difference between semi-sterile and full preparation and draping techniques have not been well reported in the literature. This is an important factor in the current state of cost savings and quality issues in surgery. Analysis at our institution found a cost savings when using semi-sterile 
technique rather than full preparation and draping, in terms of surgical supply costs and decreased preparation-to-incision time. Although this savings initially does not seem substantial, the high volume of supracondylar fractures treated yearly as well as decreased surgical time makes these factors of greater importance to the health care system.

There were some limitations in the current study. The sterility of wires, drapes, scrubbing characteristics of staff assistants, gloving techniques, operation theatre conditions, number of skin piercings by wires and patient's nutrition status were not accounted for; however, the impacts of these should assumedly be comparable regardless of which technique was used. With the low incidence of infection found, these variables did not have a notable impact. Fracture type was not recorded for 60 patients in the semi-sterile group as well as 68 patients in the full preparation and draping group. Number of k-wires and medial pins were also not charted or available on $\mathrm{x}$-ray for a small percentage of patients in each group as demonstrated in Table 1.

As this study pertained to both sexes and all skeletally immature individuals under 16 years of age, it is reasonable to assume that semi-sterile technique can be used safely to treat most supracondylar fractures with CRPP. The study findings cannot be generalized to patients with open fractures, those with additional ipsilateral arm fractures or those that are immunocompromised.

\section{Conclusion}

No difference in infection rate using semi-sterile operative technique compared to full preparation and draping in CRPP of supracondylar fractures was found in this study. The administration of pre-operative antibiotics does not appear to make a difference in infection rates. Semi-sterile operative technique is cost effective and decreases preparation-to-incision time which may be an advantage of this approach, depending on patient volumes.

\section{Declarations}

Ethics approval and consent to participate:

Ethics was attained from the University of Manitoba Research Ethics Board for this study.

The need for consent from participants in this study was waived and deemed unnecessary by the University of Manitoba Research Ethics Board.

Consent for publication:

Not Applicable.

Availability of data and materials: 
The datasets used and/or analysed during the current study are available from the corresponding author on reasonable request.

Competing interests:

The authors declare that they have no competing interests.

\section{Funding:}

Alexander Gibson Fund : Bursary to cover chart retrieval and publication costs.

Authors' Contributions:

IL - Conception, design of the work, analysis of data and manuscript writing

KS - substantial acquisition of data and analysis of data

MX - substantial acquisition of data and analysis of data

JT - substantial acquisition of data and analysis of data

SM - substantial analysis of data and manuscript editing

PJ - substantial conception, design of the work

All authors read and approved the final manuscript.

Acknowledgements:

Not applicable.

\section{Abbreviations}

CRPP

Closed reduction percutaneous pinning

CDN

Canadian

\section{References}

1. Reynolds RA, Mirzayan R (2000). A technique to determine proper pin placement of crossed pins in supracondylar fractures of the elbow. J Pediatr Orthop 20:485-9.

2. Turgut, A., Onvural, B., Kazimoglu et al (2016). How safe is the semi-sterile technique in the percutaneous pinning of supracondylar humerus fractures? Ulus Trauma Acil Cerrahi Derg,22(5):477- 
482.

3. Aubret, S., Lecointe, T., Mansour et al (2017). Risk of infection and secondary displacement in pediatric supracondylar or lateral condyle fractures treated with unburied Kirchener-wires removed before complete bone healing. Journal of Pediatric Orthopaedics B,26:222-226.

4. lobst, C., Spurdle, C., King et al (2007). Percutaneous Pinning of Pediatric Supracondylar Humerus Fractures with the Semisterile Technique. J Pediatr Orthop,27: 17-22.

5. Ponce BA, Hedequist DJ, Zurakowski D et al (2004). Complications and timing of follow-up after closed reduction and percutaneous pinning of supracondylar humerus fractures: follow-up after percutaneous pinning of supracondylar humerus fractures. J Pediatr Orthop 24:610-4.

6. Shannon FJ, Mohan P, Chacko J et al (2004). Percutaneous lateral cross-wiring of supracondylar fractures of the humerus in children. J Pediatr Orthop 24:376-9.

7. Skaggs DL, Cluck MW, Mostofi A et al (2004). Lateral-entry pin fixation in the management of supracondylar fractures in children. J Bone Joint Surg Am 86:702-7.

8. Topping RE, Blanco JS, Davis TJ (1995). Clinical evaluation of crossed-pin versus lateral-pin fixation in displaced supracondylar humerus fractures. J Pediatr Orthop. 15:435-9.

9. Bashyal RK, Chu JY, Schoenecker PL et al (2009). Complications after pinning of supracondylar distal humerus fractures. J Pediatr Orthop 29:704-708.

10. Mehlman C, Strub WM, Roy DR (2001). The effect of surgical timing on the perioperative complications of treatment of supracondylar humeral fractures in children. J Bone Joint Surg Am 83:323-327.

11. Devkota P, Khan JA, Acharya BM et al (2008). Outcome of supracondylar fractures of the humerus in children treated by closed reduction and percutaneous pinning. J Nepal Med Assoc 47:66-70.

12. Gartland JJ (1959). Management of supracondylar fractures of the humerus in children. Surg Gynecol Obstet 109:145-154.

13. Kao HK, Chen MC, Lee WC et al (2014). A prospective comparative study of pin site infection in pediatric supracondylar humeral fractures: daily pin care vs. no pin care. Arch Orthop Trauma Surg 134:919-923.

14. Shamsuddin, S., Penafort, R., \& Sharaf, I (2001). Crossed-pin versus lateral-pin fixation in pediatric supracondylar fractures. Med J Malaysia,56:38-44.

15. Parikh, S., Lykissas, M., Roshdy, M. et al (2015). Pin tract infection of operatively treated supracondylar fractures in children: long-term functional outcomes and anatomical study. $J$ Child Orthop,9:295-302. 
16. Morand, P., Billoet, A., Rottman, M et al (2009). Specific Distribution within the Enterobacter cloacae Complex of Strains Isolated from Infected Orthopedic Implants. J Clin Microbiol 47:8.

\section{Table}

Table 1

Description of patients in semi-sterile and full preparation and draping groups including complications.

\begin{tabular}{|c|c|c|c|}
\hline & Semi-sterile & $\begin{array}{l}\text { Full preparation } \\
\text { and draping }\end{array}$ & $\mathrm{p}$-value \\
\hline Male: Female & $93: 75$ & $90: 78$ & 0.74 \\
\hline Age (years; mean \pm SD) & $5.7 \pm 2.1$ & $5.9 \pm 2.4$ & 0.42 \\
\hline \multicolumn{4}{|l|}{ Fracture type (n (\%)) } \\
\hline - Type 2 & 23/108 (21\%) & $37 / 100(37 \%)$ & \\
\hline - Type 3 & $85 / 108(79 \%)$ & $63 / 100(63 \%)$ & \\
\hline \multicolumn{4}{|l|}{ Number of K-wires (n (\%)) } \\
\hline$\cdot 2$ & $66 / 142(46 \%)$ & $92 / 148(62 \%)$ & \\
\hline$\cdot 3$ & $72 / 142(51 \%)$ & $50 / 148(34 \%)$ & \\
\hline$\cdot 4$ & $4 / 142(3 \%)$ & $6 / 148(4 \%)$ & \\
\hline Medial pins used (n (\%)) & $3 / 142(0.02 \%)$ & $2 / 148(0.01 \%)$ & \\
\hline $\begin{array}{l}\text { Prep to incision time }(\min ) \\
(\text { mean } \pm S D)\end{array}$ & $2.4 \pm 2.0$ & $9.9 \pm 4.2$ & $<0.001$ \\
\hline Pre-operative antibiotics* $(\mathrm{n}(\%))$ & $0 / 168(0 \%)$ & $76 / 168(23 \%)$ & \\
\hline \multicolumn{4}{|l|}{ Complications (n (\%)) } \\
\hline Infections** & $0 / 168(0 \%)$ & $1 / 168(0.01 \%)$ & \\
\hline Early pin removal for migration & $3 / 168(1.8 \%)$ & $2 / 168(1.2 \%)$ & \\
\hline Revision due to loss of reduction & $2 / 168(1.2 \%)$ & $1 / 168(0.6 \%)$ & \\
\hline
\end{tabular}


Figures

358 Patient Charts were assessed for eligibility

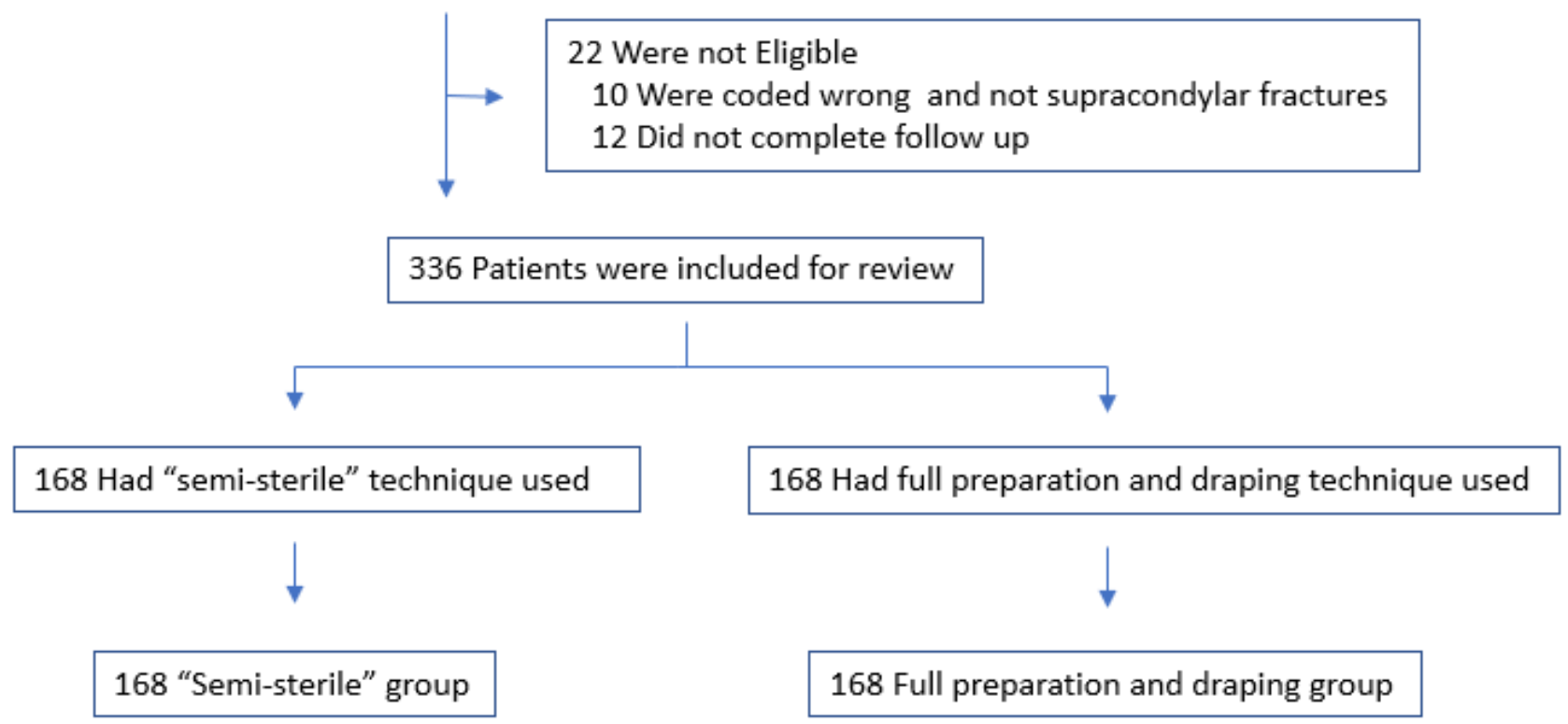

Figure 1

Flow diagram. 